\title{
Immunosuppressive treatment affects morphology and apoptotic intensity of the liver in pregnant Wistar rats
}

\author{
Dagmara Szypulska-Koziarska1, Aleksandra Wilk1, Joanna Kabat-Koperska², Agnieszka Kolasa-Wołosiuk1, \\ Kamila Misiakiewicz-Has ${ }^{1}$, Barbara Wiszniewska' ${ }^{1}$
}

'Department of Histology and Embryology, Pomeranian Medical University, Szczecin, Poland

2Department of Nephrology, Transplantology and Internal Medicine, Pomeranian Medical University, Szczecin, Poland

\begin{abstract}
Introduction: Immunosuppressive drugs are crucial for patients who have undergone transplantation. These medicines prolong proper functioning of the transplanted organ, but, on the other hand, they lead to various disorders, including pathological alterations within native organs and apoptosis. The aim of our study was to investigate the influence of immunosuppressive drugs on morphology, physiology and hepatocyte apoptosis in the liver of pregnant female Wistar rats.

Material and methods: The study was conducted on 32 female rats exposed to immunosuppressive drugs before ( 2 weeks) and during pregnancy ( 3 weeks). The regimens included drugs commonly used in therapy following kidney transplantation (cyclosporine A, mycophenolate mofetil, prednisone, tacrolimus and everolimus).

Results: We observed a decreased liver weight in all treated dams in comparison to the control rats. No statistically significant differences among all examined groups were found regarding the concentration of either alanine or aspartate aminotransferase. The most pronounced apoptosis and the most severe morphological abnormalities were found in rats treated with cyclosporine A, prednisone and everolimus; but moderate changes were visible in other treatment groups. The alterations that we observed included dilated sinusoids, inflammation foci, the disappearance of boundaries between hepatocytes, ruptured blood vessels, degradation of cytoplasm, and hemosiderosis.

Conclusions: It seems possible that the alterations seen here are due to the early stage of liver damage, and that the biochemical parameters of the liver were not affected yet. Immunosuppressive therapy during pregnancy disturbs the physiology of the liver, and causes morphological changes in this organ.

Key words: apoptotic cells, hepatocytes, immunosuppressants, pathomorphology.
\end{abstract}

Corresponding author: Aleksandra Wilk, Department of Histology and Embryology, Pomeranian Medical University, 72 Powstancow Wielkopolskich St, 70-111 Szczecin, Poland, phone: +48 502043 488, e-mail: aleksandra.wilk@pum.edu.pl 


\section{Introduction}

Following transplantation of organs, the immunological system of the recipient may engage mechanisms aimed at overcoming foreign agents, and these mechanisms can contribute to graft failure. The rejection of an allograft is mediated by the primary response of $\mathrm{T}$ lymphocytes. As a consequence we observe their infiltration of the graft and a range of inflammatory reactions. Due to the action of immunosuppressive drugs the process of organ rejection is prevented and functions of the transplanted organ are maintained. Although essential for recipients, immunosuppressive therapy can be highly hepatotoxic and nephrotoxic [1]. Immunosuppressive drugs can lead to increased apoptosis intensity in organs [2]. The elimination of unwanted cells during apoptosis occurs as a normal physiological event, but on the other hand any abnormalities in this process may result in the survival of pathological cells and their products, which can ultimately lead to the development of fibrosis in an organ [3].

Immunosuppressive therapy comprises drugs such as calcineurin inhibitors (CNI) including cyclosporine A (C) and tacrolimus (T); mTOR inhibitors (everolimus (E) and sirolimus); IMDH inhibitors including azathioprine, which is increasingly being replaced by mycophenolate mofetil (MMF); and glucocorticosteroids (G) [4-6]. These medicines are used in combined therapy, usually as a complex of three different drugs, following kidney transplantation. $\mathrm{C}$ by binding cyclophilin inhibits calcineurin and therefore leads to decreased interleukin-2 transcription and thus deactivates $\mathrm{T}$ cells [7]. It can be replaced by Tc, which acts differently in an initial pathway, as Tc binds to FK506. E inhibits proliferation of lymphocytes and synthesis of IgM, IgG and IgA by binding to FKBP and thus inhibiting mTOR kinase. E exhibits antineoplastic and antiproliferative properties such as inhibiting fibroblast proliferation and fibrosis, but it also inhibits replication of the hepatitis $\mathrm{C}$ virus (HCV) [5]. Mycophenolate mofetil is an immunosuppressive drug acting by inhibition of inosine monophosphate dehydrogenase and thus selectively inhibits lymphocyte proliferation [7]. From above-mentioned drugs E and MMF are contraindicated during pregnancy [5].

In our study, we used a combination of immunosuppressive drugs, both indicated and contraindicated during pregnancy, investigated their influence on the intensity of apoptosis in hepatocytes and determined morphological and biochemical changes in the liver of female Wistar rats treated with these drugs during pregnancy.

\section{Material and methods}

\section{Animals and treatment}

The study was conducted on 32 female Wistar rats and eight male Wistar rats for mating purposes only (all from the Centre of Experimental Medicine, Bialystok Medical University, Poland). At the start of the experiment, the rats were 12 weeks old and their mean weight was $230 \mathrm{~g}$. The animals had genetic and health certificates issued by a veterinarian. This study was approved by the Szczecin Local Ethical Committee for Experiments on Animals (No. 12/2013, dated 24 Oct 2013). All the procedures involving animals were performed in accordance with the ethical standards of the institution or practice at which the studies were conducted. The animals were housed individually on a 12-hour lightdark cycle and were given feed Labofeed H (Morawski, Kcynia, Poland) and water ad libitum. The experiment was performed using the pharmaceutical form of each drug. The animals received drugs by a stomach tube at a dose volume of $5 \mathrm{ml} / \mathrm{kg}$ every $24 \mathrm{~h}$. The doses of medicaments for rats were calculated to be an adequate equivalent as found in human therapy. Furthermore, the metabolic differences were also taken into consideration. The doses used in the study were as follows: $4 \mathrm{mg} / \mathrm{kg} /$ day T (Prograf, Astellas); $20 \mathrm{mg} / \mathrm{kg} /$ day MMF (CellCept, Roche); $5 \mathrm{mg} / \mathrm{kg} /$ day C (Sandimmun Neoral, Novartis); $0.5 \mathrm{mg} / \mathrm{kg} /$ day E (Certican, Novartis); and $4 \mathrm{mg} / \mathrm{kg} /$ day G (Encorton, Polfa). The drug doses were based on data available in the literature [8-13] to reach the level within the therapeutic range. The study model is presented in Table I. The animals received medication every $24 \mathrm{~h}$ for approximately 5 weeks ( 2 weeks prior to mating, when placed with males $1: 1$ in separate cages, and later during 3 weeks of pregnancy). After mating, each pregnant female rat was housed in a separate cage. Once a week, the pregnant animals were weighed again and the medication dose was adjusted to the new weight. After delivery, the treatment was stopped. No drugs were administered during lactation, as in humans, breastfeeding is not advised while taking immunosuppressive drugs. Thirty-one female rats completed the study, and 69 pups from the control group, 13 from the CMG group and only 1 pup from the CEG group were born. The dams were sacrificed at weaning, on day 21 after delivery. We did not sacrifice

Table I. Study model

\begin{tabular}{|l|c|c|c|c|c|}
\hline Parameter & Cyclosporin A (C) & Tacrolimus (T) & Everolimus (E) & $\begin{array}{c}\text { Mycophenolate } \\
\text { mofetil (M) }\end{array}$ & $\begin{array}{c}\text { Glucocortico- } \\
\text { steroids }(\mathrm{G})\end{array}$ \\
\hline Control group $(n=7)$ & - & - & - & - & - \\
\hline CMG group $(n=8)$ & + & - & - & + & + \\
\hline TMG group $(n=8)$ & - & + & - & + & + \\
\hline CEG group $(n=8)$ & + & - & + & - & + \\
\hline
\end{tabular}

Abbreviations of drugs used to identify the study groups: CMG - cyclosporine A + mycophenolate mofetil + glucocorticosteroids; TMG - tacrolimus + mycophenolate mofetil + glucocorticosteroids; CEG - cyclosporine A + everolimus + glucocorticosteroids. 
them earlier, as their offspring had to stay alive for other studies. The female rats were sacrificed by sodium pentobarbital (Polpharma, Poland) injection administered intraperitoneally at $40 \mathrm{mg} / \mathrm{kg}$ body weight. Body weights were measured. Blood samples were obtained from the heart for laboratory tests (aspartate aminotransferase (AST), alanine aminotransferase (ALT)). Subsequently, necropsies of all rats were performed. The livers were collected, weighed, and partly fixed in $4 \%$ buffered formalin solution for histological examination, before being partly placed in liquid nitrogen and stored at $-80^{\circ} \mathrm{C}$ before testing for markers of liver injury analysis.

\section{Histological evaluation and its criteria}

For the morphological analysis, serial slides $(3 \mu \mathrm{m})$ were stained with the PAS method. Next, the slides underwent general histological examination to investigate potential acute or chronic changes within the liver. The samples were independently examined by two experienced histologists by light microscopy (Leica).

\section{Apoptosis assessment}

In order to identify apoptotic cells, rabbit polyclonal antibody anti-active caspase-3 (R\&D Systems, Abingdon, UK, cat. no. AF835, final dilution $1: 400$ ) was used. The deparaffinized sections were microwaved in citrate buffer ( $\mathrm{pH}$ 6.0) for heat-induced epitope retrieval. After slow cooling to room temperature, the slides were washed in PBS twice for $5 \mathrm{~min}$ and then incubated for $60 \mathrm{~min}$ with the antibody. Next, slides were stained with a labeled polymer peroxidase system with $5,5^{\prime}$-diaminobenzidine (DAB) as the chromogen (EnVision + System-HRP, code K4010, DakoCytomation, Glostrup, Denmark) in accordance with the manufacturer's staining protocol. The sections were washed in distilled water and counterstained with hematoxylin. For the negative control, specimens were processed in the absence of the primary antibody. Positive staining was defined microscopically (Leica DM5000B, Wetzlar, Germany) by visual identification of brown pigmentation of the nucleus of the hepatocytes.

The percentage of apoptotic cells was calculated by the following steps: Initially, all of the hepatocytes from an equal area of the classical lobule (objective magnification $100 \times$ ) were counted (parameter A ). Secondly, the number of positively stained nuclei of hepatocytes was counted from the same area (parameter B). In the next stage parameter A was assessed to be $100 \%$, according to which parameter B was calculated as the percentage of apoptotic hepatocytes. These steps were repeated in 6 randomly chosen microphotographs from 6 rats.

\section{Statistical analysis}

The values of quantitative variables were compared between groups using nonparametric tests (Kruskal-Wallis and the Mann-Whitney $U$-test), as most of the data were not normally distributed as assessed by the Shapiro-Wilk test. The arithmetic mean $(x)$, standard deviation $( \pm S D)$, median, minimum and maximum values were calculated for each group. The cut-off level of statistical significance was set at $p<0.05$. Calculations were performed using Statistica 12 software (StatSoft, Krakow, Poland).

\section{Results}

The results of the research and the statistical analysis are presented in Tables II-IV and Figures 1-4.

Table II. Liver weight of female rats in control and treatment groups

\begin{tabular}{|l|c|c|c|c|c|}
\hline Liver mass [g] & $\begin{array}{c}\text { Control group } \\
(n=7)\end{array}$ & $\begin{array}{c}\text { CMG group } \\
(n=8)\end{array}$ & $\begin{array}{c}\text { TMG group } \\
(n=8)\end{array}$ & $\begin{array}{c}\text { CEG group } \\
(n=8)\end{array}$ & $\begin{array}{c}P \text { (Kruskal-Wallis } \\
\text { test) }\end{array}$ \\
\hline$x \pm \mathrm{SD}$ & $12.05 \pm 1.4$ & $\begin{array}{c}10.16 \pm 2.59^{*} \\
\text { vs. Control }\end{array}$ & $\begin{array}{c}9.64 \pm 1.08^{*} \\
\text { vs. Control }\end{array}$ & $\begin{array}{c}9.48 \pm 0.69^{* *} \\
\text { vs. Control }\end{array}$ & 0.026 \\
\hline Median & 12.2 & 9.4 & 9.65 & 9.73 & $8.3-10.2$ \\
\hline Range & $10.4-13.6$ & $7.7-15.9$ & $8.0-10.9$ & 8 & \\
\hline
\end{tabular}

Kruskal-Wallis test value indicates statistical significance at $p<0.05 . x$ - arithmetic mean, $\mathrm{SD}$ - standard deviation, $p$ - level of significance, $\mathrm{CMG}-\mathrm{cyclosporine} \mathrm{A}+$ mycophenolate mofetil + glucocorticosteroids; TMG - tacrolimus + mycophenolate mofetil + glucocorticosteroids; CEG -cyclosporine A + everolimus + glucocorticosteroids; ${ }^{*} p<0.05,{ }^{* *} p<0.01$ (Mann-Whitney test).

Table III. AST and ALT levels of female rats in control and treatment groups

\begin{tabular}{|c|c|c|c|c|c|c|}
\hline \multicolumn{2}{|l|}{ Parameter } & $\begin{array}{l}\text { Control group } \\
\qquad(n=7)\end{array}$ & $\begin{array}{l}\text { CMG group } \\
\quad(n=8)\end{array}$ & $\begin{array}{l}\text { TMG group } \\
\quad(n=8)\end{array}$ & $\begin{array}{l}\text { CEG group } \\
(n=8)\end{array}$ & $\begin{array}{c}P \text { (Kruskal-Wallis } \\
\text { test) }\end{array}$ \\
\hline \multirow[t]{3}{*}{ AST [U/I] } & $x \pm \mathrm{SD}$ & $154.83 \pm 60.73$ & $197.33 \pm 87.23$ & $161.75 \pm 43.94$ & $184.29 \pm 59.95$ & 0.62 \\
\hline & Median & 148 & 163 & 155.5 & 169 & \\
\hline & Range & 85-228 & $128-369$ & $112-248$ & $98-275$ & \\
\hline \multirow[t]{3}{*}{ ALT [U/I] } & $x \pm \mathrm{SD}$ & $75.66 \pm 13.53$ & $81 \pm 12.88$ & $84.25 \pm 29.88$ & $90.57 \pm 19.52$ & 0.65 \\
\hline & Median & 79.5 & 81.5 & 79.5 & 91 & \\
\hline & Range & $49-86$ & $65-99$ & $37-137$ & $66-119$ & \\
\hline
\end{tabular}

Kruskal-Wallis test value indicates statistical significance at $p<0.05 . x$ - arithmetical mean, $\mathrm{SD}-$ standard deviation, $p-$ level of significance, $\mathrm{CMG}-\mathrm{cyclosporine} \mathrm{A}+\mathrm{my}-$ cophenolate mofetil + glucocorticosteroids; TMG - tacrolimus + mycophenolate mofetil + glucocorticosteroids; CEG -cyclosporine A + everolimus + glucocorticosteroids 
Table IV. Apoptosis intensity in hepatocytes of livers of female rats in control and treatment groups

\begin{tabular}{|l|c|c|c|c|c|}
\hline Variable & $\begin{array}{c}\text { Control group } \\
(n=7)\end{array}$ & $\begin{array}{c}\text { CMG group } \\
(n=8)\end{array}$ & $\begin{array}{c}\text { TMG group } \\
(n=8)\end{array}$ & $\begin{array}{c}\text { CEG group } \\
(n=8)\end{array}$ & $\begin{array}{c}P \\
\text { (Kruskal-Wallis test) }\end{array}$ \\
\hline $\mathrm{X} \pm \mathrm{SD}$ & $0.39 \pm 0.22$ & $0.8 \pm 0.25$ & $0.82 \pm 0.15$ & $0.997 \pm 0.005$ & 0.001 \\
\hline Median & 0.32 & $0.98 *$ vs. CEG & $\begin{array}{c}0.84^{*} \text { vs. CEG, } \\
* *\end{array}$ & $1.0^{* *}$ vs. Control & \\
\hline Range & $0.22-0.8$ & $0.35-1.0$ & $0.56-0.99$ & $0.99-1.0$ & \\
\hline
\end{tabular}

Kruskal-Wallis test value indicates statistical significance at $p<0.05, x$ - arithmetic mean, SD - standard deviation, $p-$ level of significance, CMG - cyclosporine A + mycophenolate mofetil + glucocorticosteroids, TMG - tacrolimus + mycophenolate mofetil + glucocorticosteroids, CEG - cyclosporine A + everolimus + glucocorticosteroids, ${ }^{*} p<0.05$ (Mann-Whitney $U$ test), ${ }^{* *} p<0.001$ (Mann-Whitney $U$ test).

\section{Liver weight}

Significantly lower weights of the livers in all treatment groups of rats compared to the control dams were observed. Furthermore, the most pronounced difference was noted between CEG and control groups (Table II).

\section{ALT and AST concentrations}

No significant differences in AST or ALT concentrations were noted among examined groups in comparison to the control dams (Table III).

\section{Apoptosis assessment}

The apoptotic intensity was significantly higher in TMG and CEG groups in comparison to the control group. Fur-
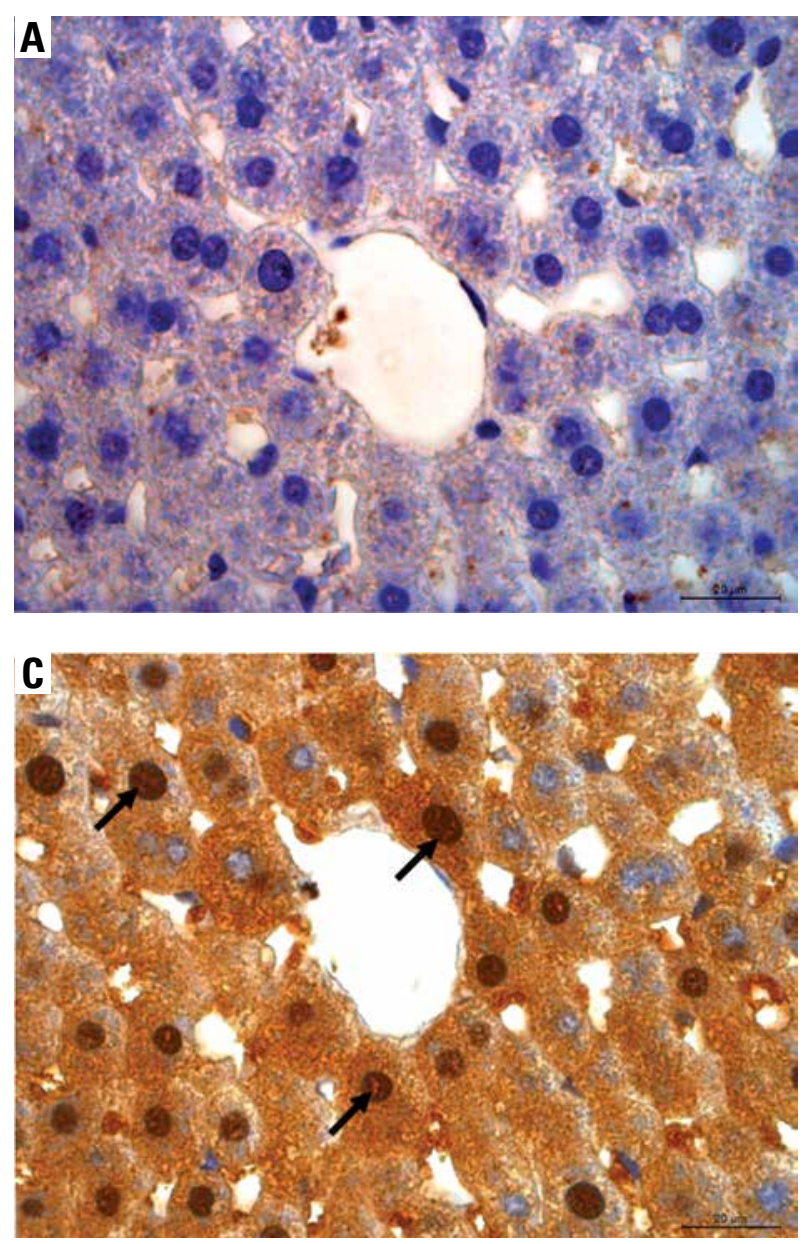

thermore, among all of the treatment groups the most pronounced apoptotic intensity was noted in the CEG group compared with CMG and TMG groups (Figure 1, Table IV).

\section{Histopathological evaluation of the liver}

We observed a number of abnormalities among the groups of dams treated with different immunosuppressive drugs. In the livers of rats of the CMG group, dilatation and congestion of blood sinusoids were seen. What is more, distortion of the hepatocytes' cytoplasm due to fat accumulation in large numbers of hepatocytes was evident. Occasionally small inflammation foci in the hepatic stroma were observed (Figure 2). In the livers of rats from the TMG group, we noted similar alterations to those of animals from
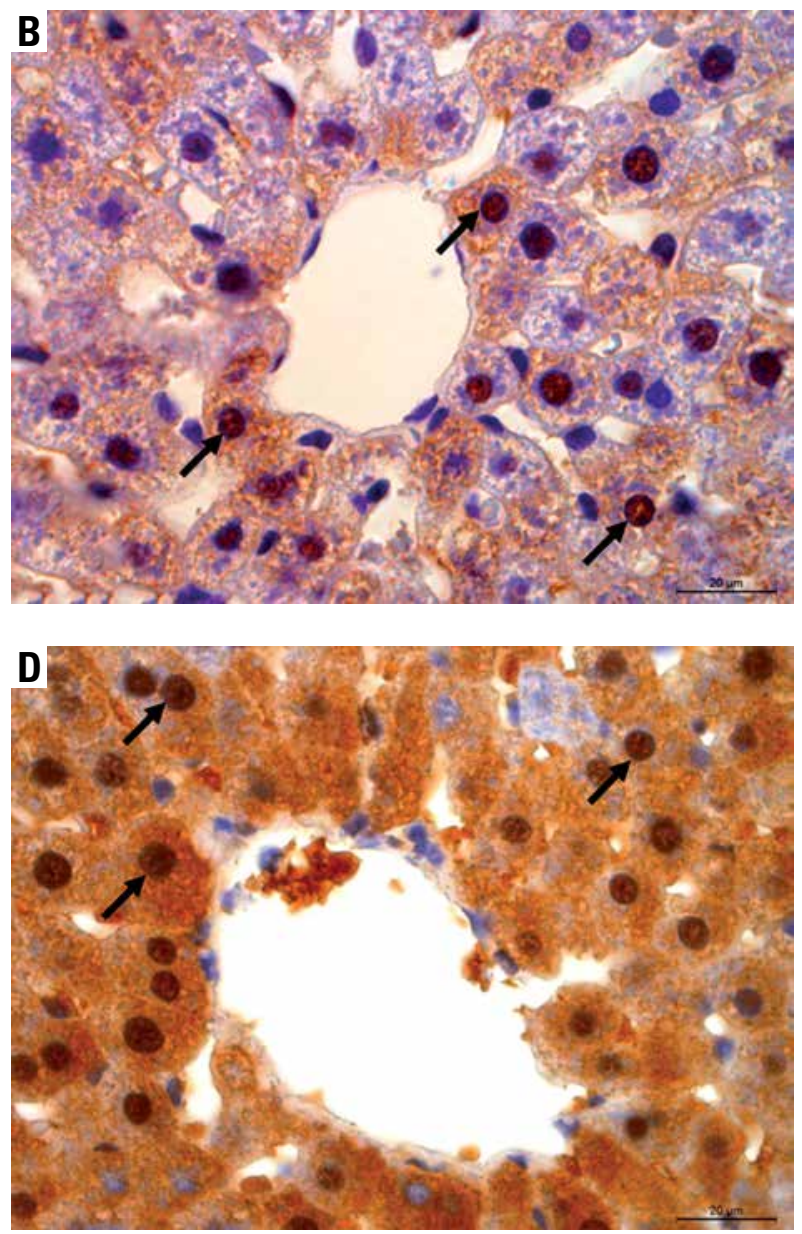

Figure. 1. Image of liver from control rats (A) and rats from treated groups (B - CMG, $\mathbf{C}-\mathrm{TMG}, \mathbf{D}-\mathrm{CEG})$ immunohistochemically stained to show expression of caspase 3. Black arrows show an example of positive (brown) staining apoptotic nucleus. Scale bar $=$ $20 \mu \mathrm{m}$. Objective magnification $100 \times$ 
the CMG group, although dilated and congested sinusoids were seen with higher frequency and the degree of dilation was higher. In some of the hepatocytes, we also observed the disappearance of the boundaries between hepatic cells, which could correspond to ruptures of blood vessels and the distortion of the cytoplasm (Figure 3). In dams from the CEG group, small inflammatory foci were seen, as was the emptying of the hepatocytes' cytoplasm due to fat accumulation in large numbers of hepatocytes. In a high number of hepatocytes edema was evident; these cells were highly swollen and filled with lipid droplets. Occasionally, in cells adjacent to highly swollen and degenerated hepatocytes, dividing hepatocytes in the stage of metaphase were noted. Highly dilated sinusoids were often visible and ruptured sinusoids were sometimes present. In the livers of dams of the CEG group, we also observed the presence of granules of hemosiderin, but the granules were only visible under higher magnification (Figure 4).

\section{Discussion}

The model of treatment studied here reflects the immunosuppressive therapy commonly used in clinical practice in humans after kidney transplantation. The used doses of drugs allowed a therapeutic concentration to be obtained in
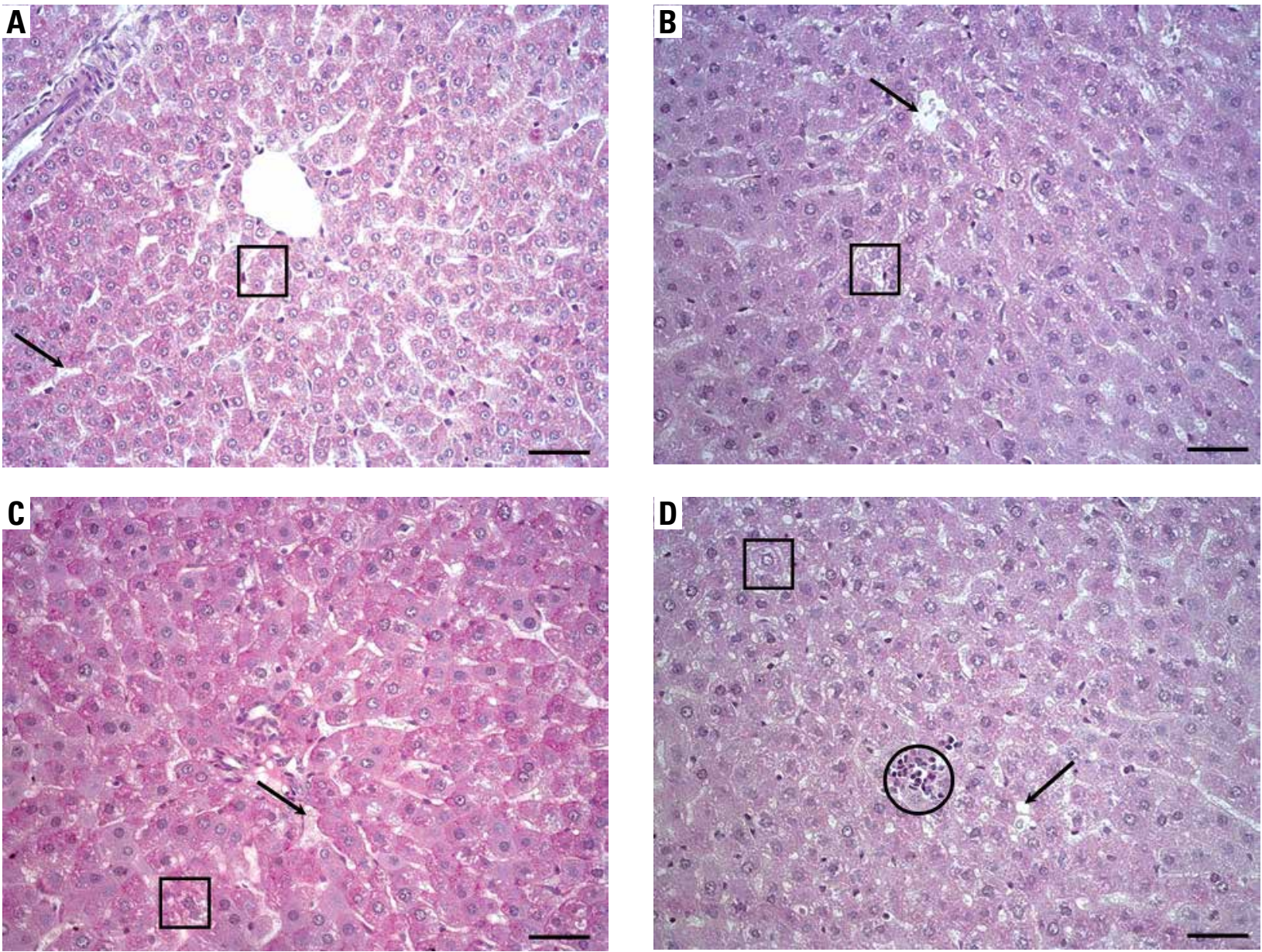

Figure. 2. Histological structure of liver of rats from the CMG group. Dilated and congested hepatic sinusoids (arrow) are visible. In a large number of hepatocytes distortion and degeneration of the hepatocyte cytoplasm due to fat accumulation (a square frame) are evident. Occasionally small inflammation foci (circle) are present. PAS staining. Scale bar $=50 \mu \mathrm{m}$. Objective magnification: $40 \times$ 
be that the liver is more vulnerable than the kidney to the impact of immunosuppressive drugs.

The findings in this study revealed the highest concentration of AST in the CMG group and the highest concentration of ALT in the CEG group, but the differences were not statistically significant. It can therefore be concluded that liver function, including the synthesis of enzymes, was not affected. These findings are thus contrary to those of Korolczuket al. [20], where mature male rats were exposed to $\mathrm{C}$ at a dose of $15 \mathrm{mg} /$ day subcutaneously for 28 days and exhibited significantly increased concentrations of both AST and ALT. The increase of ALT and AST [20] was probably caused by the three-times higher dose of $\mathrm{C}$ used, compared to our study. Other authors have obtained results similar to those of Korolczuk et al. [20-25]. It is worth noting that, in all of these experiments, the animals were exposed only to a single immunosuppressive drug, C.

Preparations made from livers of rats stained with the PAS method were evaluated to find potential histopathological changes. In our study, we observed numerous alterations between the groups of dams treated with different immunosuppressive drugs. Dilated congested sinusoids were observed in the livers of the rats of the CMG group. This finding may indicate that immunosuppressive drugs are able to
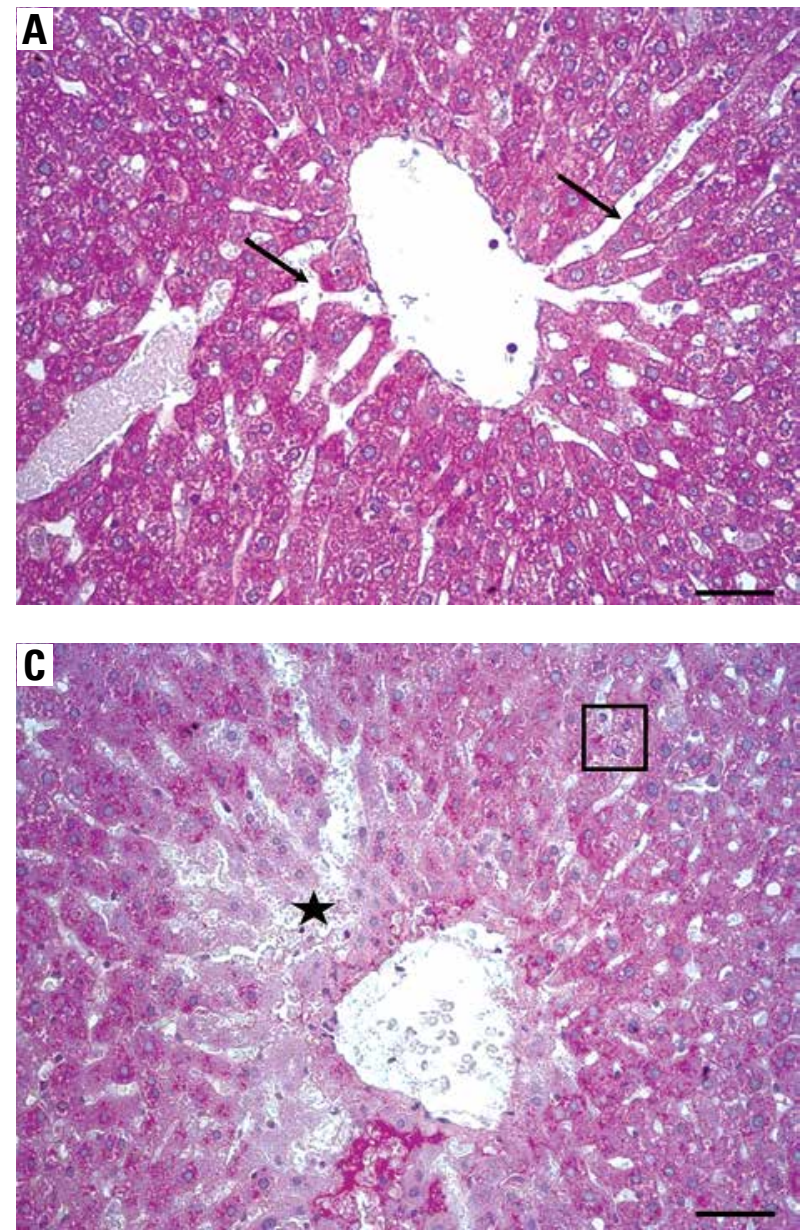

interfere with endothelial cells of particular blood vessels, thus affecting their permeability. Distortion and swelling of a large number of hepatocytes were also seen in CMG dams. It seems possible that the edema of hepatocytes results from affected permeability of their cell membranes. Furthermore, the cloudy swelling might be correlated with the disturbed membrane function and membrane transport, thus leading to a massive influx of water and sodium ions [26]. The emptying of hepatocyte cytoplasm leading to the appearance of its spongy structure in large numbers of hepatocytes and, occasionally, small inflammation foci were seen. Such alterations, apart from the emptying of the hepatocyte cytoplasm, were seen rather rarely.

Dams from the TMG group were characterized by more pronounced pathomorphological changes than in the CMG rats, and we additionally observed the disappearance of boundaries between some of the hepatocytes. These findings were correlated with ruptured blood vessels and degradation of the cytoplasm.

The most severe histopathological alterations were evaluated in the dams of the CEG group, where not only above-mentioned alterations were seen with higher frequen$\mathrm{cy}$, but the presence of hemosiderin granules was also evident. Our results correlate with others [20,27].
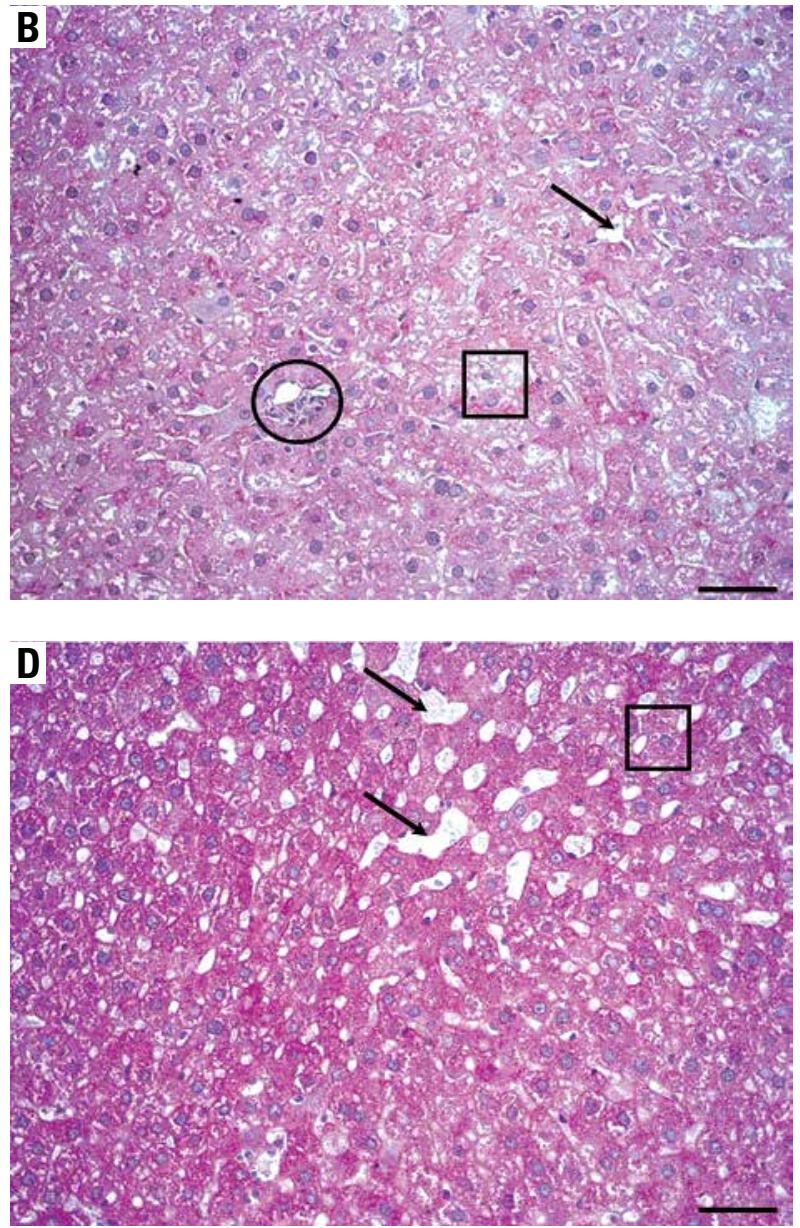

Figure. 3. Histological structure of liver of rats from the TMG group. Dilated and congested sinusoids (an arrow) are visible with high frequency. In some of the hepatocytes the disappearance of the boundaries between hepatocytes corresponding to ruptures of blood vessels (star) is observed. Distortion of the hepatocyte cytoplasm due to fat accumulation (square frame), and small inflammation foci (circle) are visible. PAS staining. Scale bar $=50 \mu \mathrm{m}$. Objective magnification: $40 \times$ 
As the concentrations of ALT and AST in the serum were not significantly affected, it seems likely that these histopathological changes were not sufficiently severe to affect the proper function of the liver. This may also be related to the efficient repair mechanisms of the liver, which were able to compensate for the damaging effects of immunosuppressive therapy $[2,16]$. Perhaps in the case of longer periods of immunosuppressive therapy, the physiological condition of the liver would also be affected [2].

Apoptosis is an important process which eliminates unwanted cells in order to prevent the survival of pathological cells and fibrosis of an organ. Under physiological conditions, this complex process is the result of the disintegration of the cell membrane and is followed by a reduction in cell size, shrinking of the cell, cytoplasmic vacuolization, disintegration of the genetic material, and finally equal fragmentation of chromatin [2]. Nevertheless, many of the above concepts are not relevant to apoptosis in pathobiology - for example, during immunosuppressive therapy, since pathological apoptosis involves large number of cells, is nonselective, can be sustained over time, and often occurs in an inflammatory milieu. Lipid products from apoptotic cells can serve as chemotactic factors and recruit inflammatory cells; thus the physiological concept that apoptosis in hepatocytes is innocuous cannot be transferred to pathological apoptosis [3]. Our findings clearly show more pronounced apoptosis in the livers of dams from the TMG and CEG groups than in the control rats ( $p=0.0007$ and $p=0.001$, respectively). What is more, we also observed significant differences between the treatment groups; the most pronounced apoptosis was seen in the CEG group in comparison to both the TMG $(p=0.04)$ and CMG group $(p=0.04)$. Perhaps E exhibits the most harmful effect on apoptosis, since Kędzierska et al. [16] confirmed that sirolimus, which belongs to the same group of drugs as $\mathrm{E}$, administered together with $\mathrm{C}$, affects the oxidoreductive balance in human endothelial cells (in vitro) much more intensely than any other immunosuppressive drug complex. In a previous study from the same experiment concerning renal tissue, more pronounced apoptosis in the proximal nephron tubules in dams from treatment groups was observed [2].

\section{Conclusions}

We observed many histopathological changes in the livers of dams treated with immunosuppressive drugs, compared with the control group; the apoptosis index in these groups was meaningful. Additionally, the most pronounced
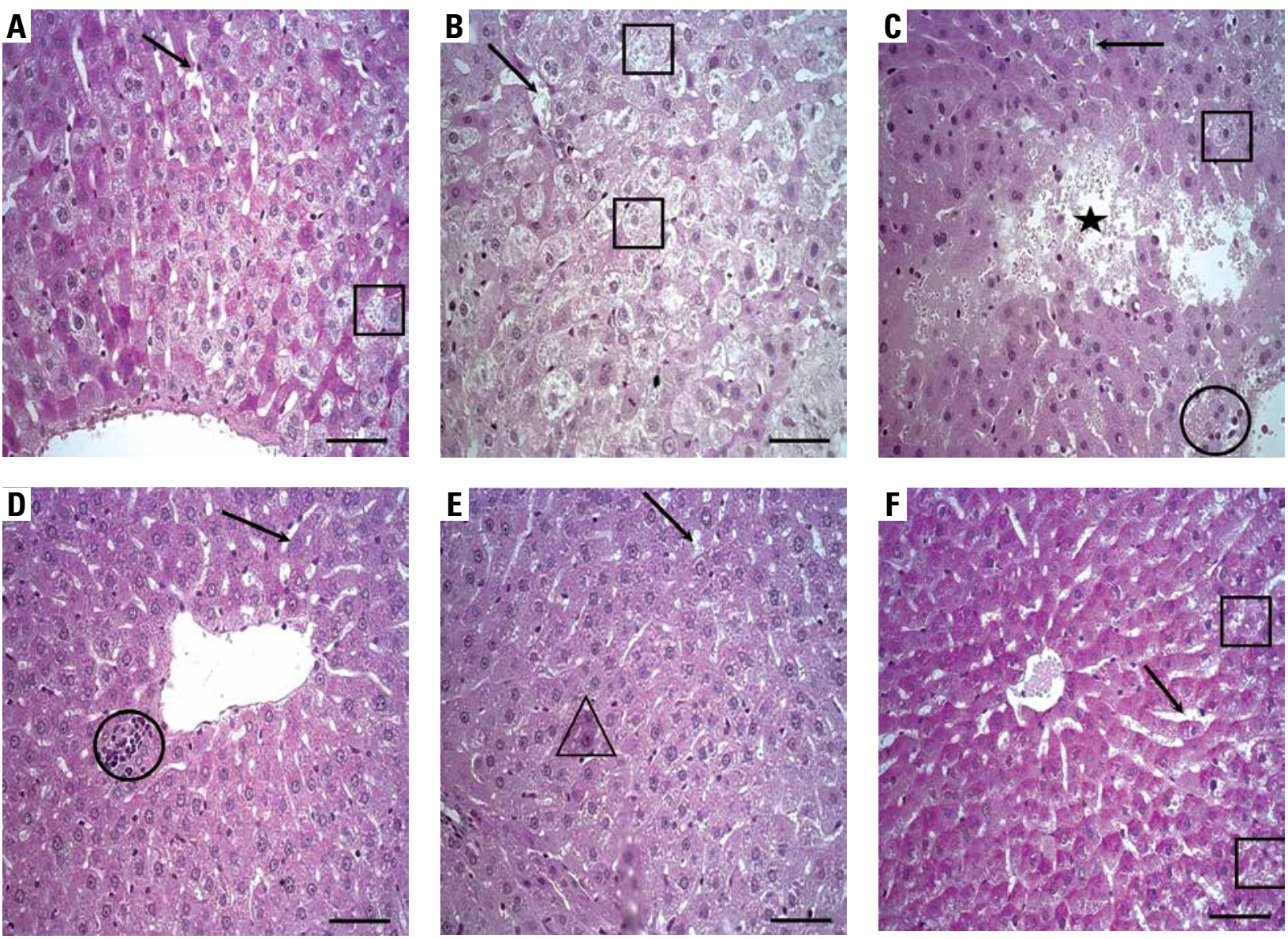

Figure. 4. Histological structure of liver of rats from the CEG group. Small inflammation foci (circle) and emptying of the hepatocyte cytoplasm (square frame) due to fat accumulation in large numbers of hepatocytes are evident. Highly dilated sinusoids (arrow) and ruptured blood vessels (star) are present. Occasionally presence of hemosiderin granules of single hepatocytes (triangular frame) is noticed, although the hemosiderin granules are only visible under higher magnification. PAS staining. Scale bar $=50 \mu \mathrm{m}$. Objective magnification: $40 \times$ 
apoptotic intensity was found in dams from the CEG group, which correlates with the most severe morphological alterations in that group. Similarly, in CMG rats, the intensity of apoptosis was the lowest, which also corresponds with the least severe morphological alterations. It seems possible that the alterations seen here are due to the early stage of liver damage, and that the biochemical parameters of the liver were not affected yet. Immunosuppressive therapy during pregnancy disturbs the physiology of the liver, and causes morphological changes in this organ.

\section{Conflict of interest}

The authors declare no conflict of interest.

\section{References}

1. Ferjanih H, El Arem A, Bouranoui A, et al. Protective effect of mycophenolate mofetil against nephrotoxicity and hepatotoxicity induced by tacrolimus in Wistar rats. J Physiolbiochem 2016; 72: 133-44.

2. Kędzierska K, Sindrewicz K, Sporniak-Tutak K, et al. Does immunosuppressive therapy affect markers of kidney damage? Ann Transplant 2016; 21: 137-44.

3. Lim EJ, Chin R, Nachbur U, et al. Effect of immunosuppressive agents on hepatocyte apoptosis post-liver transplantation. PLoS One 2015; 10: e0138522.

4. Kim YJ, Lee ES, Kim SH, et al. Inhibitory effects of rapamycin on the different stages of hepatic fibrosis. World J Gastroenterol 2014; 20: 7454-60.

5. Hwang S, Ahn CS, Kim KH, et al. A cross-sectional analysis of long-term immunosuppressive regimens after liver transplantation at Asian medical center: increased preference for mycophenolate mofetil. Ann Hepatobiliary Pancreat Surg 2018; 22: 19-26.

6. Ma Y, Kobayashi T, Kuzuya T, et al. Is absorption profile of cyclosporine really important for effective immunosuppression? Biol Pharm Bull 2006; 29: 336-342.

7. Freitas KM, Almeida JM, Monteiro JC, et al. The effects of cyclosporin A and Heteropterys tomentosa on the rat liver. An Acad Bras Cienc 2015; 87: 369-79.

8. Zhao N, Yang S, Jia Y, et al. Maternal betaine supplementation attenuates glucocorticoid-induced hepatic lipid accumulation through epigenetic modification in adult offspring rats. J Nutr Biochem 2018; 54: 105-12.

9. Kua AH, Li J, Li C, et al. Prenatal steroid administration leads to adult pericardial and hepatic steatosis in male baboons. Int J Obes 2017; 41: 1299-302.

10. Piao SG, Bae SK, Lim SW, et al. Drug interaction between cyclosporine and mTOR inhibitors in experimental model of chronic cyclosporine nephrotoxicity and pancreatic islet dysfunction. Transplantation 2012; 93: 383-9.

11. Rovira J, Marcelo Arellano E, Burke JT, et al. Efffect of mTOR inhibitor on body weight: from an experimental rat model to human transplant patients. Transpl Int 2008; 21: 992-8.

12. Sagiroglu T, Sezer A, Torun N, et al. Protective effect of everolimus on renal ischemia reperfusion injury in rats. Saudi J Kidney Dis Transpl 2014; 25: 294-302.

13. Schmitz V, Klawitter J, Bendrick-Peart J, et al. Metabolic profiles in urine reflect nephrotoxicity of sirolimus and cyclosporine fol- lowing rat kidney transplantation. Nephron Exp Nephrol 2009; 111: 80-91.

14. Kabat-Koperska J, Kolasa-Wołosiuk A, Baranowska-Bosiacka I, et al. The influence of exposure to immunosuppressive treatment during pregnancy on renal function and rate of apoptosis in native kidneys of female Wistar rats. Apoptosis 2016; 21: 1240-8.

15. Kędzierska K, Sporniak-Tutak K, Sindrewicz K, et al. Effect of immunosuppressive treatment on protein expression in rat kidney. Drug Des Devel Ther 2014; 8: 1695-708.

16. Kędzierska K, Sporniak-Tutak K, Kolasa-Wołosiuk A, et al. The effect of immunosuppressive therapy on renal cell apoptosis in native rat kidneys. Histol Histopatol 2015; 30: 105-16.

17. Lloberas N, Torras J, Alperovich G, et al. Different renal toxicity profiles in the association of cyclosporine and tacrolimus with sirolimus in rats. Nephrol Dial Translplant 2008; 23: 3111-9.

18. Neiashabouri SH, Hutson SM, Davoodi J. Chronic activation of mTOR complex 1 by branched chain amino acids and organ hypertrophy. Amino Acids 2015; 47: 1167-82.

19. Gielchinsky Y, Laufer N, Weitman E, et al. Pregnancy restores the regenerative capacity of the aged liver via activation of an mTORC1-controlled hyperplasia/hypertrophy switch. Genes Dev 2010; 24: 543-8.

20. Korolczuk A, Caban K, Amarowicz M, Czechowska G, Irla-Miduch J. Oxidative stress and liver morphology in experimental cyclosporine A-induced hepatotoxicity. Biomed Res Int 2016; 2016 : 5823271.

21. Erarlsan E, Ekiz F, Uz B, et al. Effect of erdosteine on cyclosporine-induced hepatotoxicity in rats. Drug Chem Toxicol 2011; 34: 32-7.

22. Erdem SR, Emre-Aydingoz S, Atilla P, et al. Cyclosporine-A- induced acute hepatotoxicity in guinea pigs in associated with endothelium-mediated decrease in local hepatic blood flow. Life Sci 2011; 88: 753-60.

23. Kaya H, Koc A, Sogut S, et al. The protective effect of N-acetylcysteine against cyclosporine A-induced hepatotoxicity in rats. J Appl Toxicol 2008; 28: 15-20.

24. Kurus M, Esrefoglu M, Karabulut AB, Sogutlu G, Kaya M, Otlu A. Oral 1-arginine protects against cyclosporine-induced hepatotoxicity in rats. Exp Toxicol Pathol 2008; 60: 411-9.

25. Pari L, Sivasankari R. Effect of elagic acid on cyclosporine-induced oxidative damage in the liver of rats. Fundam Clin Pharmacol 2008; 22: 395-401.

26. Alarifi S, Ali D, Al Doaiss AA, Ali BA, Ahmed M, Al-Khedhairy AA. Histologic and apoptotic changes induced by titanium dioxide nanoparticles in the livers of rats. Int J Nanomed 2013; 8: 3937-43.

27. Battino M, Bompadre S, Leone L, et al. The effect of cyclosporine-A chronic administration on the antioxidant pattern of rat liver mitochondria: structural and functional consequences. Bio Factors 2003; 18: 271-5. 